\title{
Ionic Liquid Derived Ultrafine Pt Nanoparticles with Uniformly Dispersed State for Long-Life Oxygen Electroreduction
}

\author{
Qi Li, ${ }^{*} \uparrow$ Shizhu Song, ${ }^{\dagger}$ and Yanqing Wang $*,+$ \\ †Department of Materials Science and $\$$ Department of Chemical Engineering, School \\ of Chemistry and Chemical Engineering, Nantong University, Nantong 226019, \\ Jiangsu, P. R. China
}

Total number of pages (including cover): 17

Total number of tables: 1

Total number of figures: 18

Supporting references: 18 


\section{Contents}

1. SEM and TEM images................................................... 2

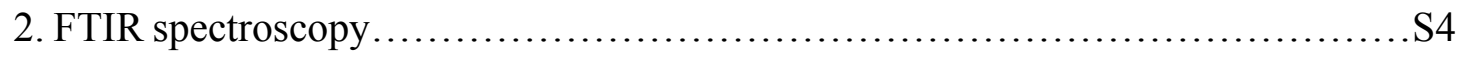

3. Photographs....................................................... S5

4. ${ }^{1} \mathrm{H}$ proton nuclear magnetic resonance spectroscopy.......................... 5

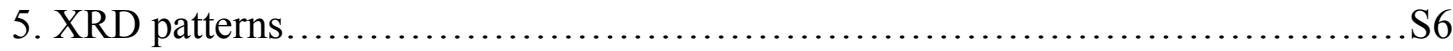

6. Energy dispersive X-ray (EDX) spectroscopy............................ S7

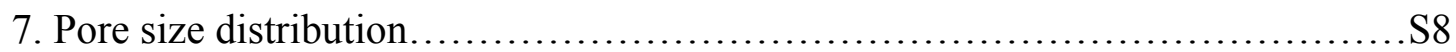

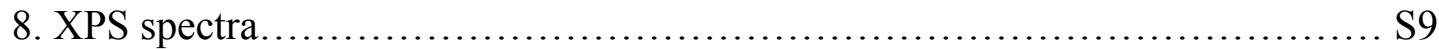

9. Electrochemical tests................................................... S11

10. References.......................................................... S14 


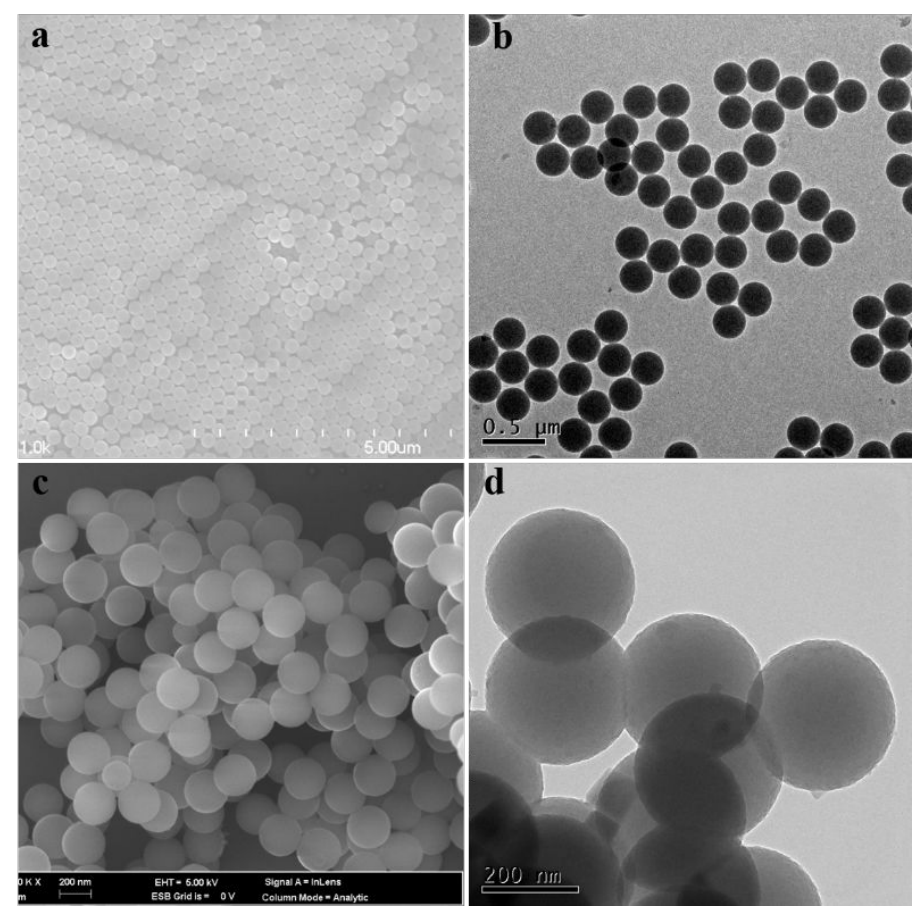

Figure S1. (a) SEM and (b) TEM images of PS nanopsheres. (c) SEM and (d) TEM images of PS@PPy composite.

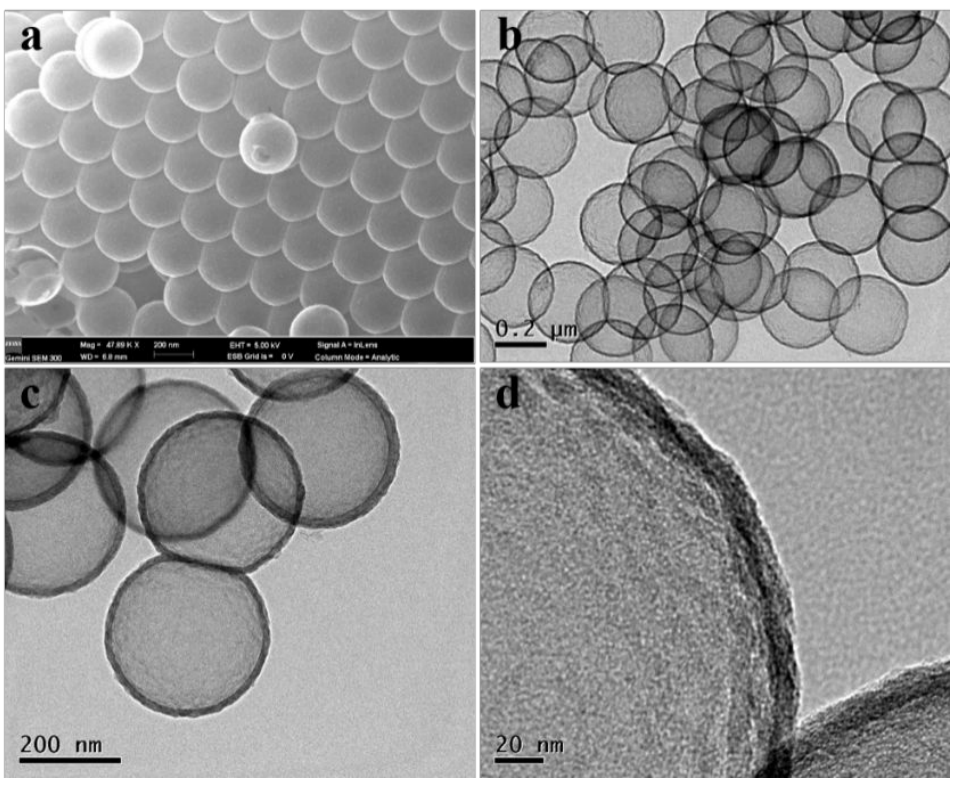

Figure S2. (a) SEM and (b), (c), (d) TEM images of HCNs, respectively. 

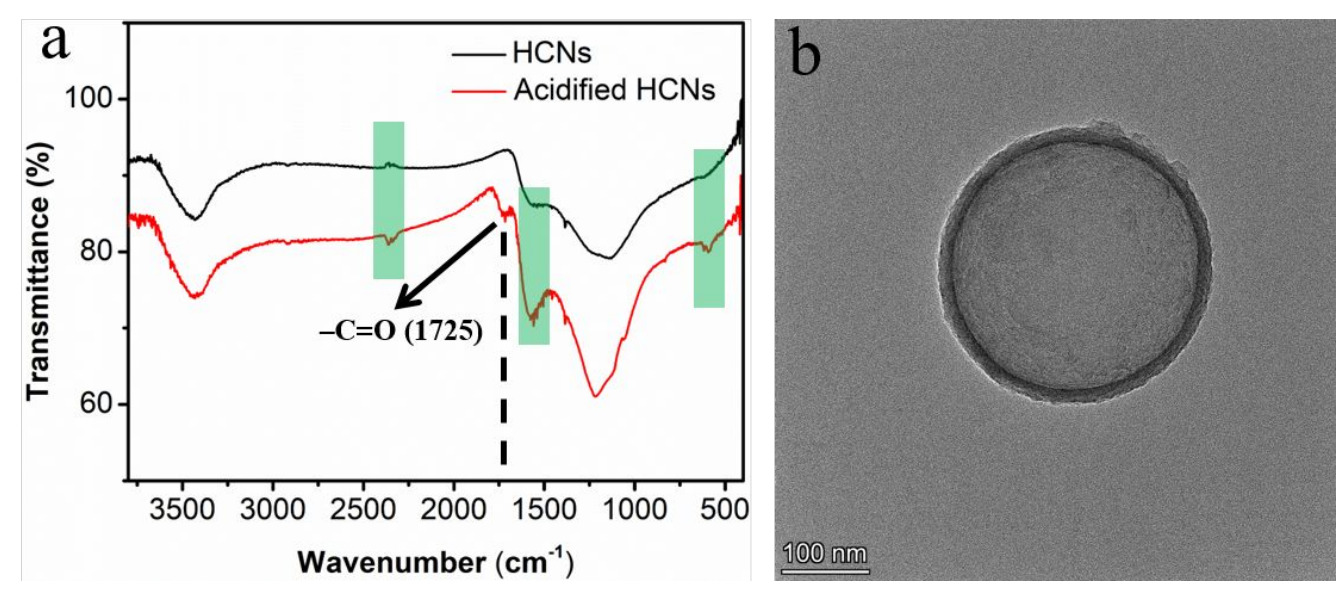

Figure S3. FTIR spectroscopy and TEM image of the acidified HCNs.

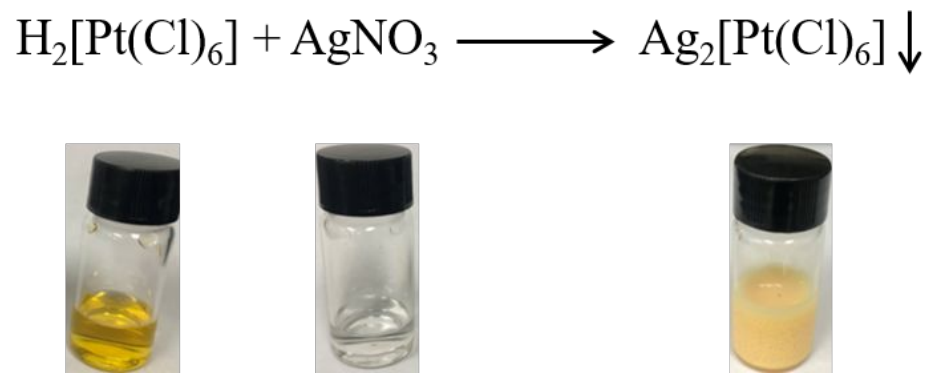

Figure S4. Photographs of chloroplatinic acid, silver nitrate, and silver chloroplatinate. 


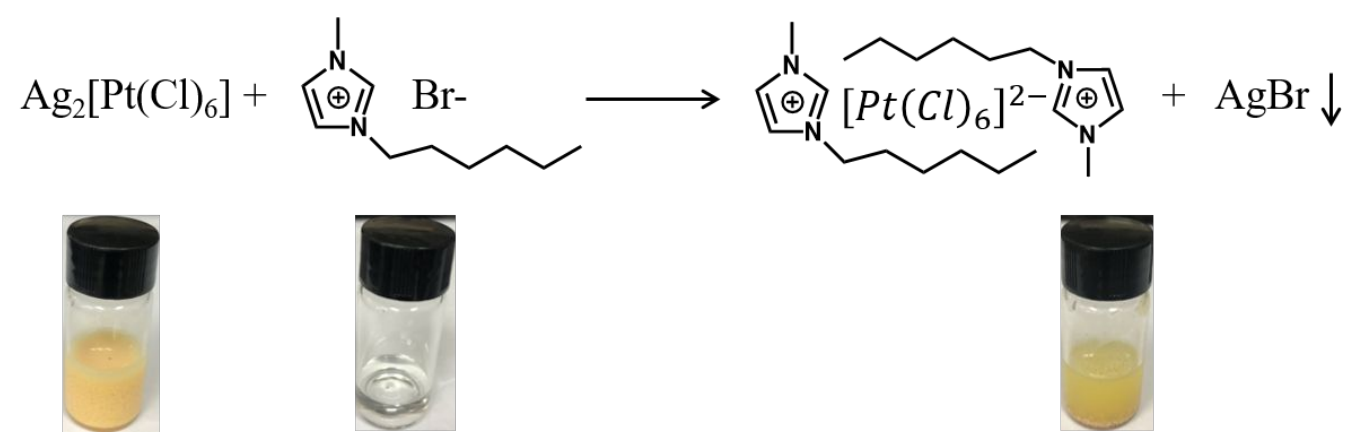

Figure S5. Photographs of silver chloroplatinate, 1-methyl-3hexyl-imidazolium bromide, and reaction production between silver chloroplatinate and 1-methyl-3hexylimidazolium bromide.

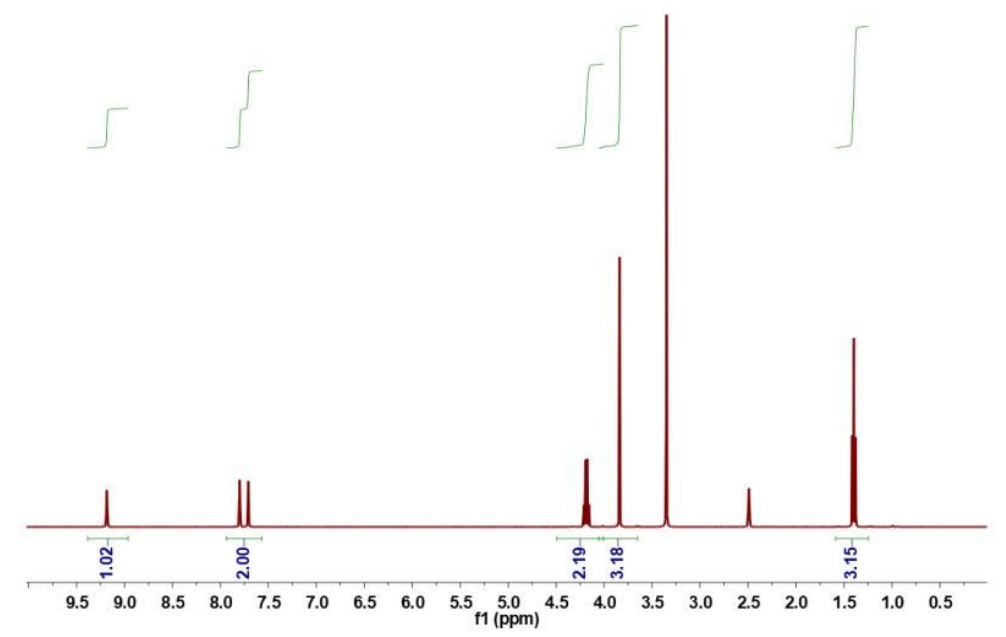

Figure S6. ${ }^{1} \mathrm{H}$ proton nuclear magnetic resonance spectroscopy of the 1-hexyl-3methylimidazolium- $\left[\mathrm{Pt}(\mathrm{Cl})_{6}\right]^{2-}$ ionic liquid. 

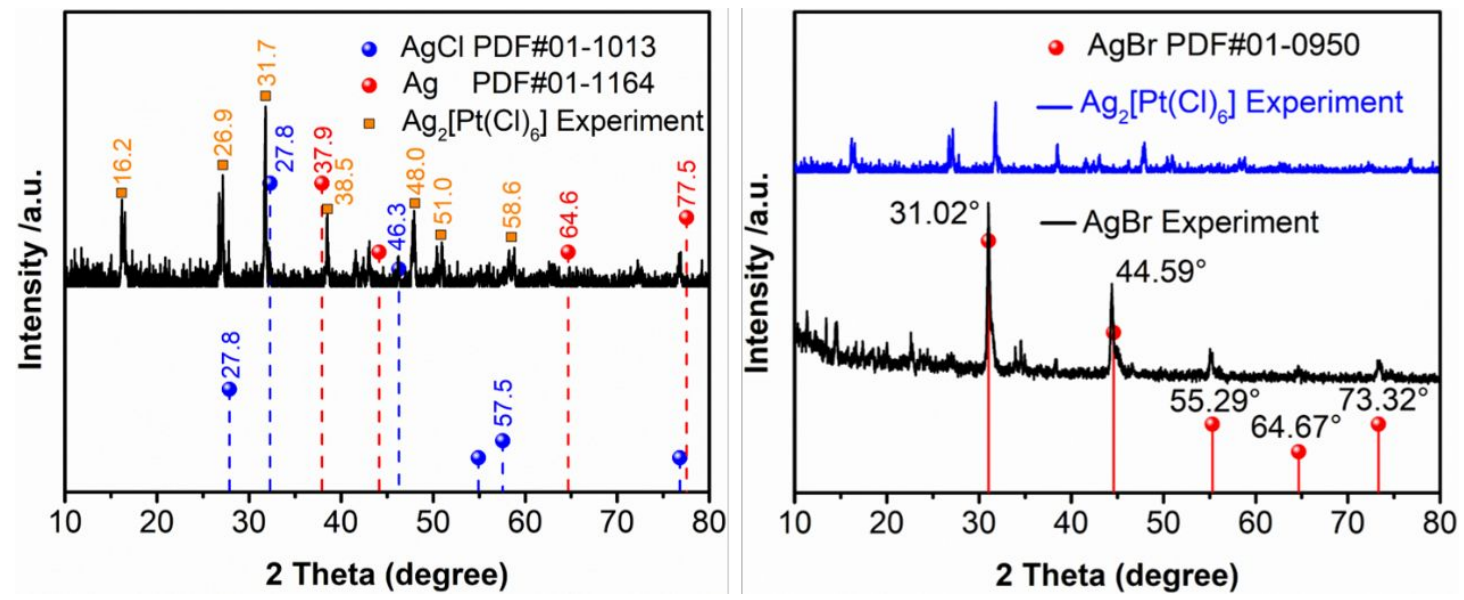

Figure S7. XRD patterns of the $\mathrm{Ag}_{2}\left[\mathrm{Pt}(\mathrm{Cl})_{6}\right]$ and $\mathrm{AgBr}$ precipitates obtained from the anion exchange reaction.

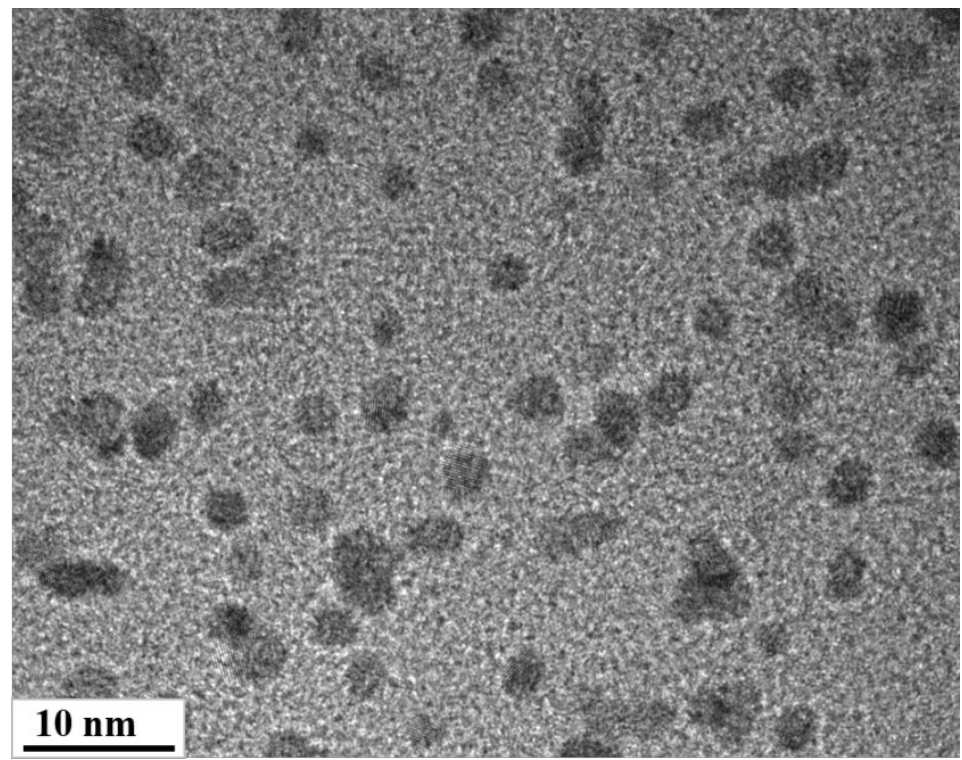

Figure S8. Large magnification TEM image of $\mathrm{Pt}_{\mathrm{IL}}-\mathrm{HCNs}$. 


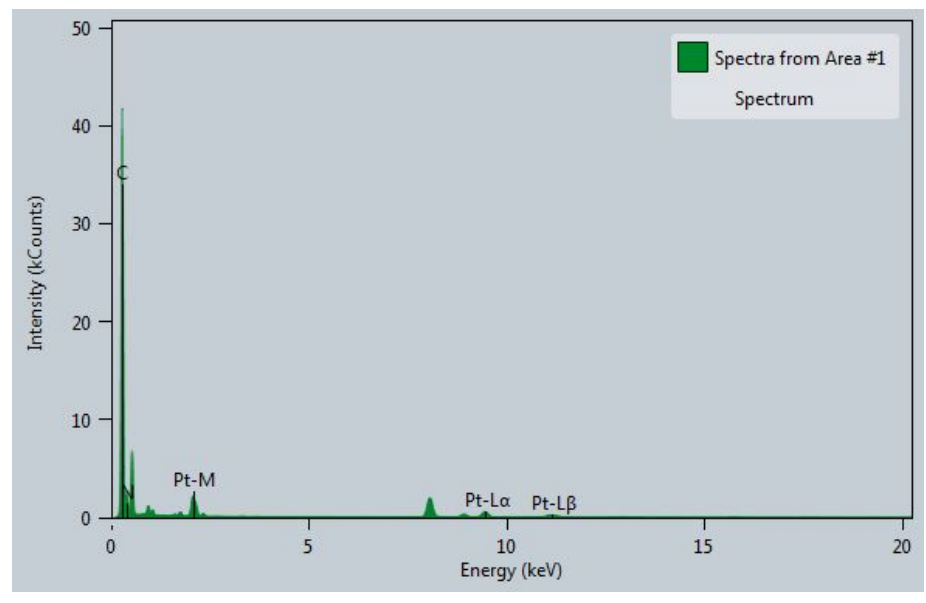

Figure S9. Scanning transmission electron microscope (STEM)-energy dispersive Xray $(\mathrm{EDX})$ spectroscopy of the $\mathrm{Pt}_{\mathrm{IL}}$-HCNs.

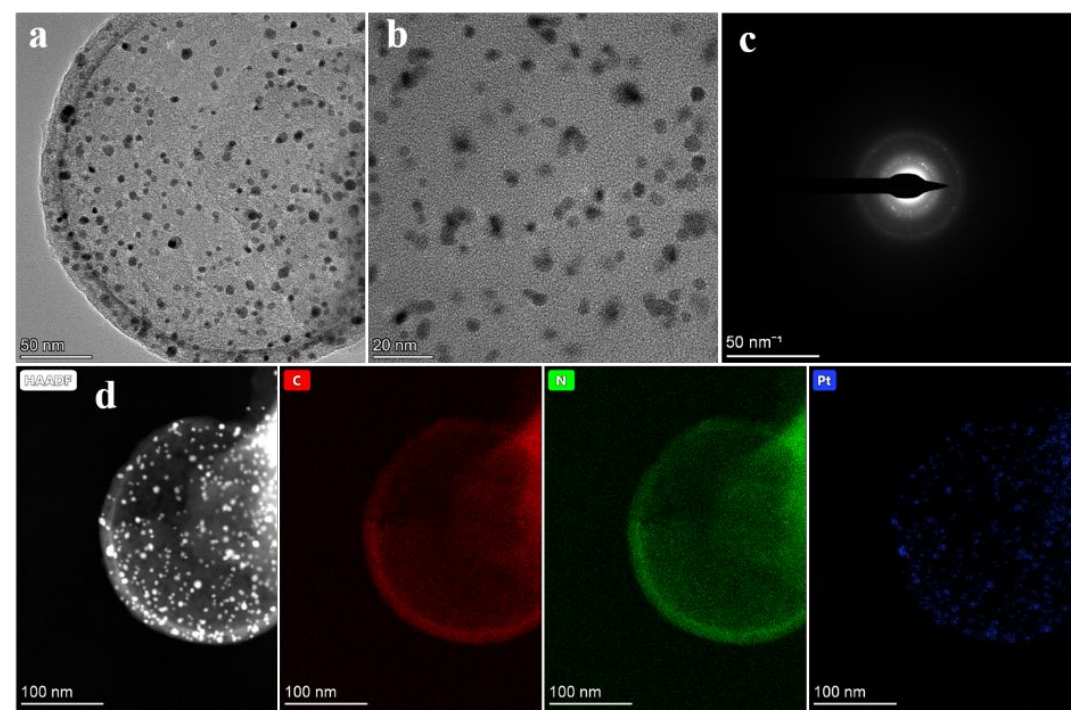

Figure S10. The morphology characterization of Pt-HCS. (a, b) TEM image, (c) SAED pattern, (d) HAADF-STEM image and corresponding elemental mapping of $\mathrm{C}, \mathrm{N}$, and Pt. 


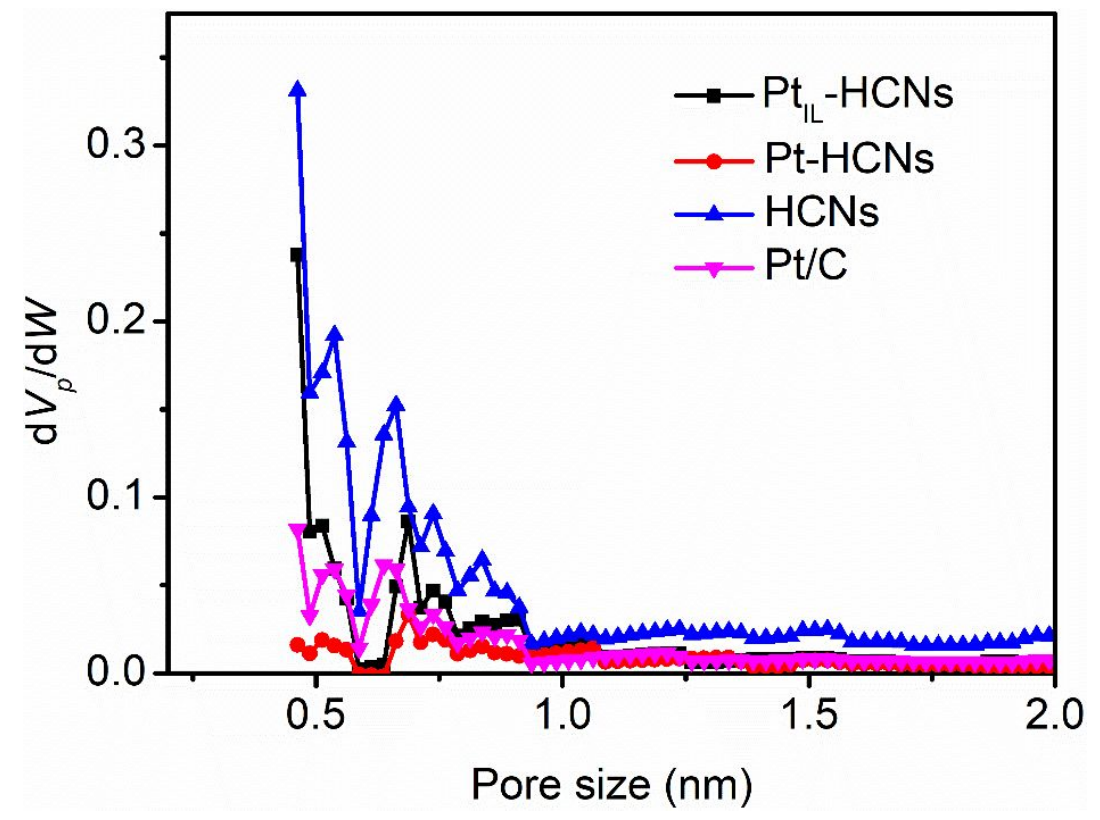

Figure S11. Pore size distribution of $\mathrm{Pt}_{\mathrm{IL}}-\mathrm{HCNs}$, Pt-HCNs, HCNs, and Pt/C.

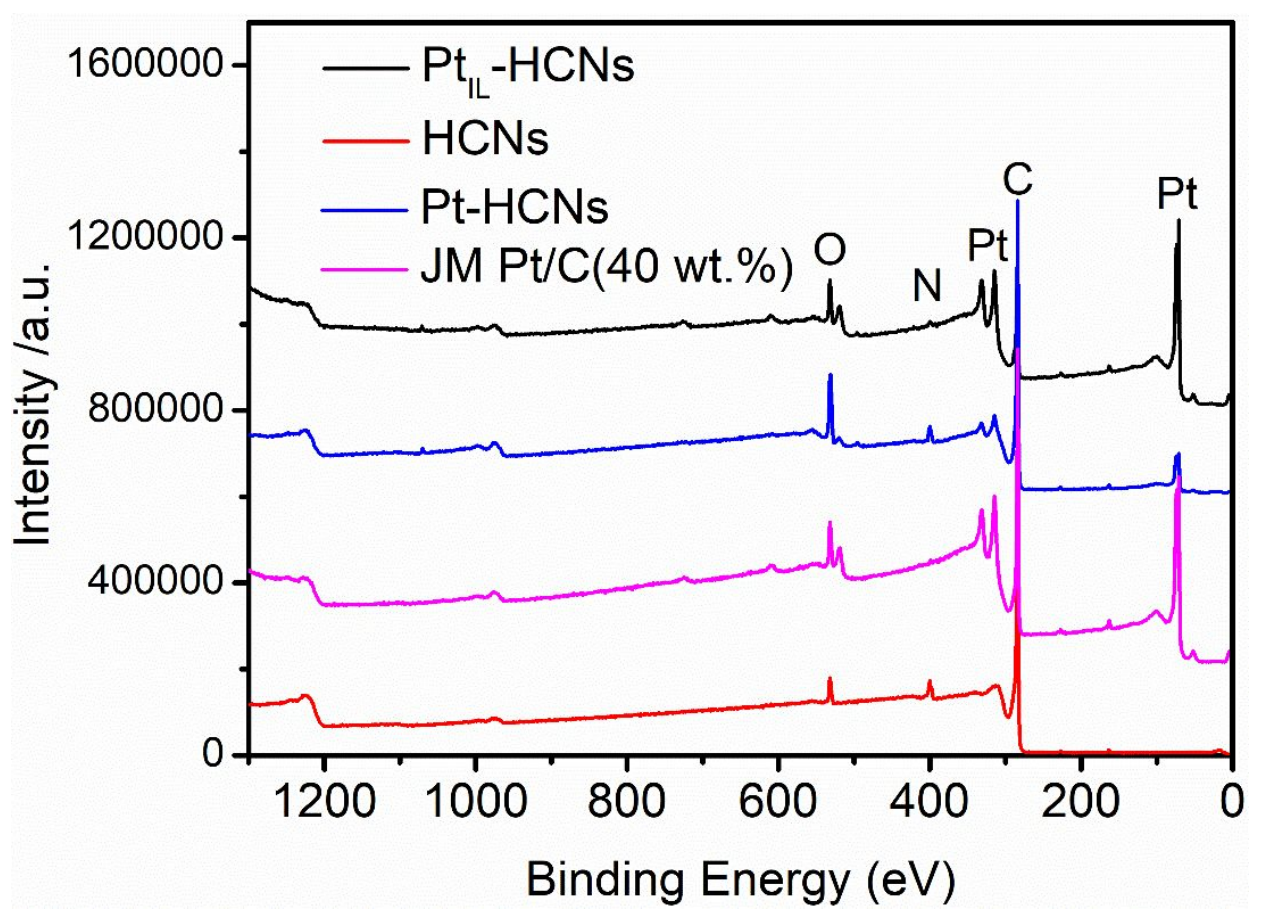

Figure S12. XPS survey spectra of $\mathrm{Pt}_{\mathrm{IL}}-\mathrm{HCNs}$, Pt-HCNs, HCNs, and Pt/C. 

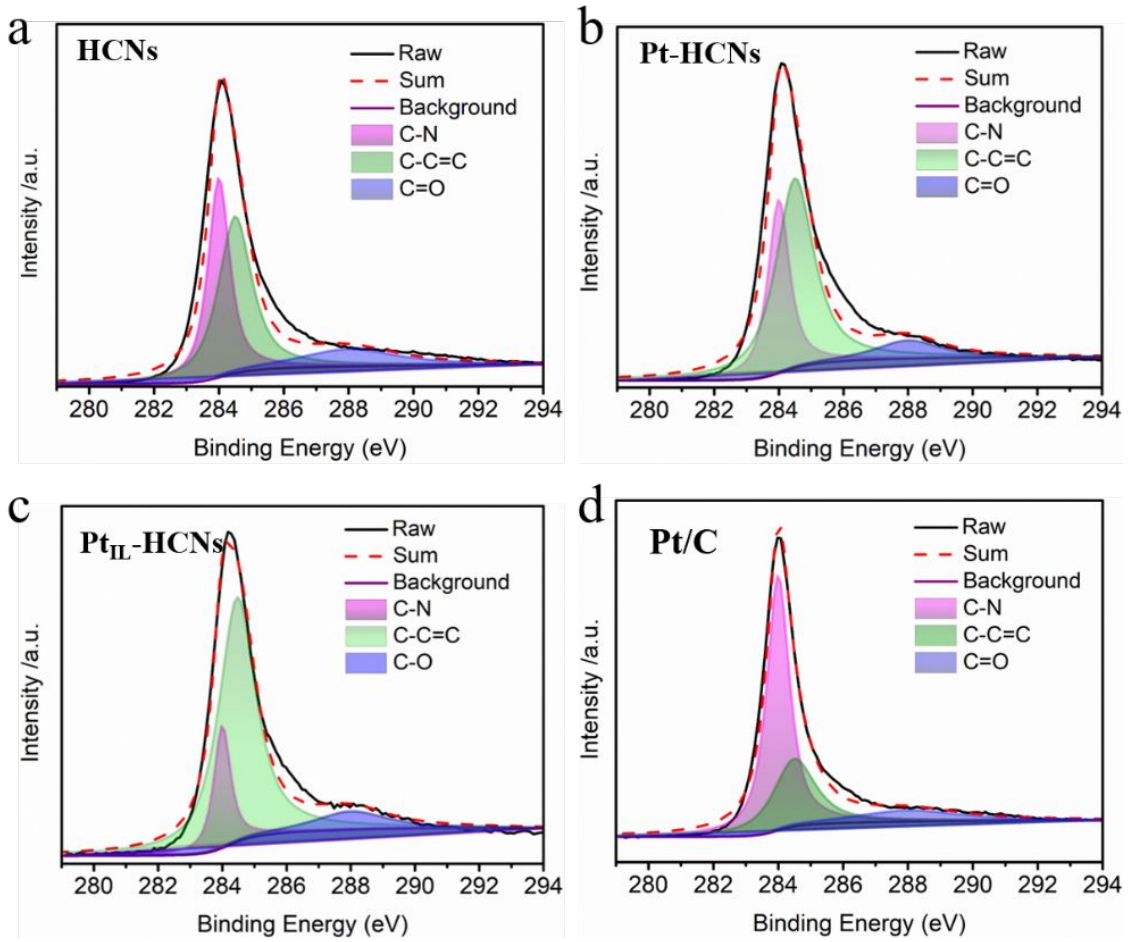

Figure S13. High-resolution C1s XPS spectra of (a) $\mathrm{Pt}_{\mathrm{IL}}-\mathrm{HCNs}$, (b) Pt-HCNs, (c) HCNs, and (d) Pt/C. 

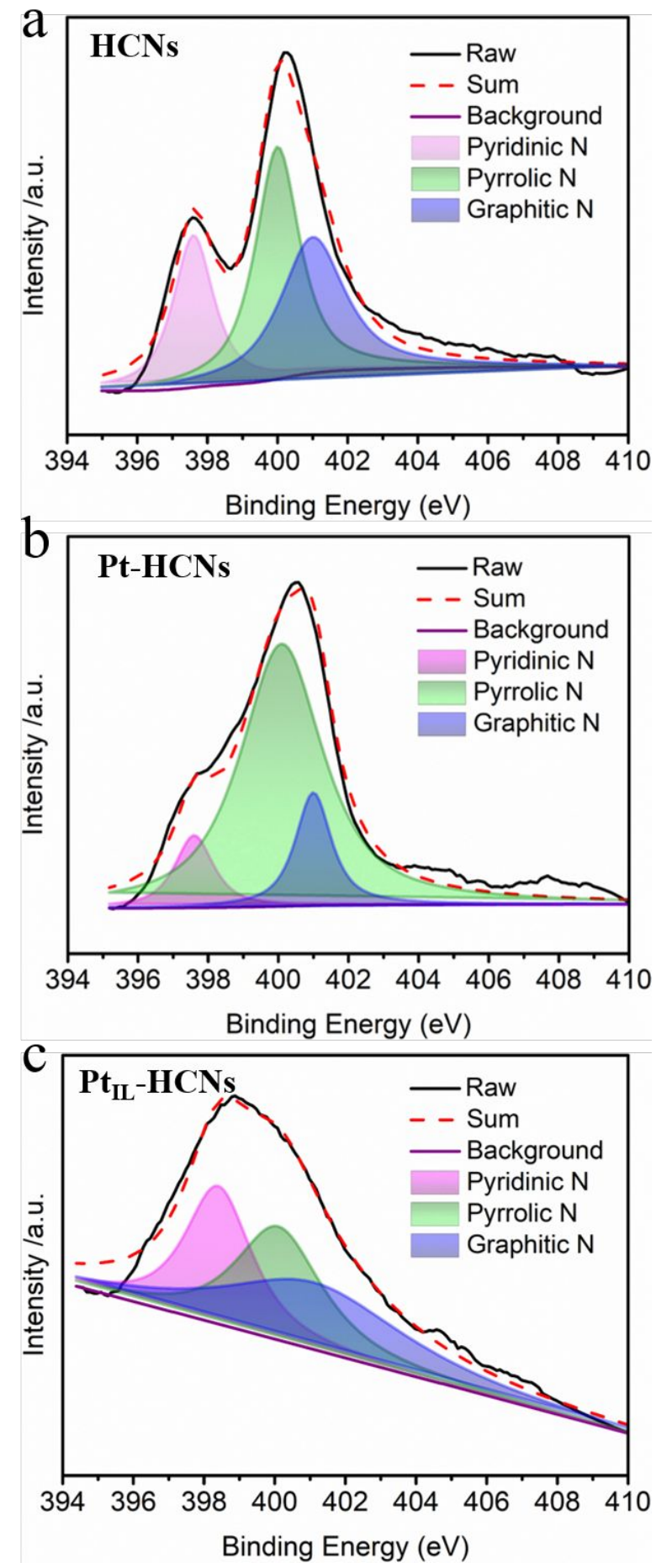

Figure S14. High-resolution N1s XPS spectra of (a) $\mathrm{Pt}_{\mathrm{IL}}-\mathrm{HCNs}$, (b) Pt-HCNs, and (c) HCNs. 

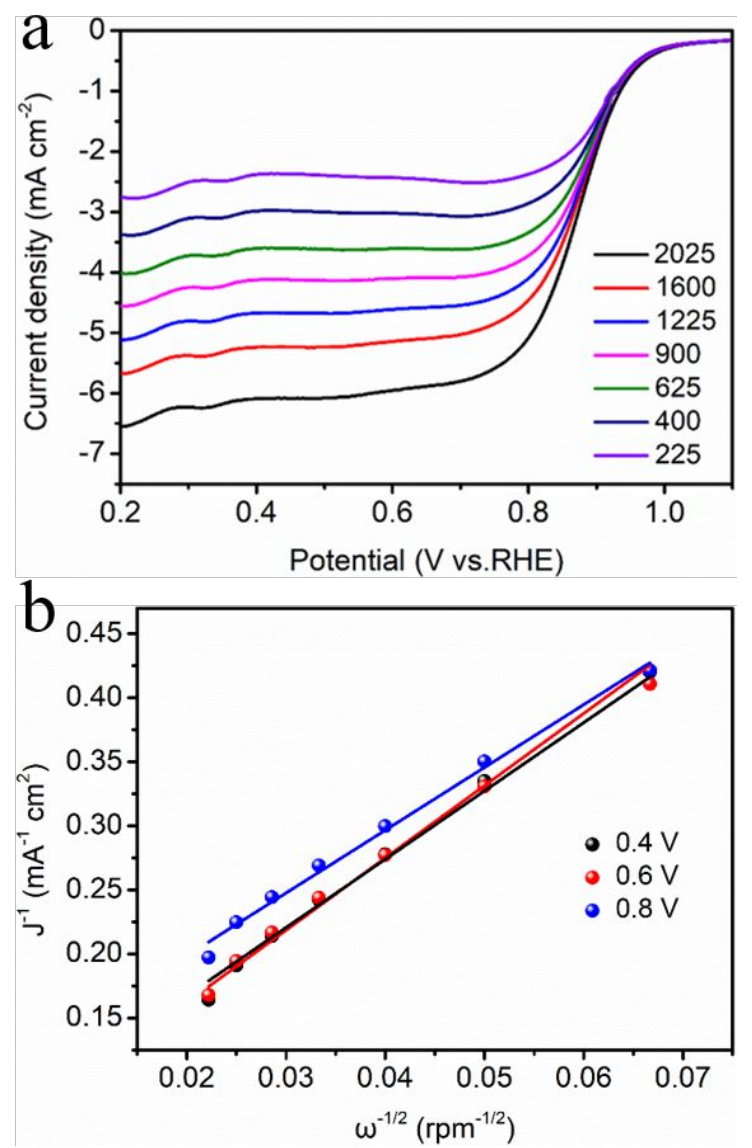

Figure S15. (a) Polarization curves of $\mathrm{Pt}_{\mathrm{IL}}-\mathrm{HCNs}$ in $\mathrm{O}_{2}$-saturated $0.1 \mathrm{M} \mathrm{KOH}$ solution and (b) corresponding K-L plots.

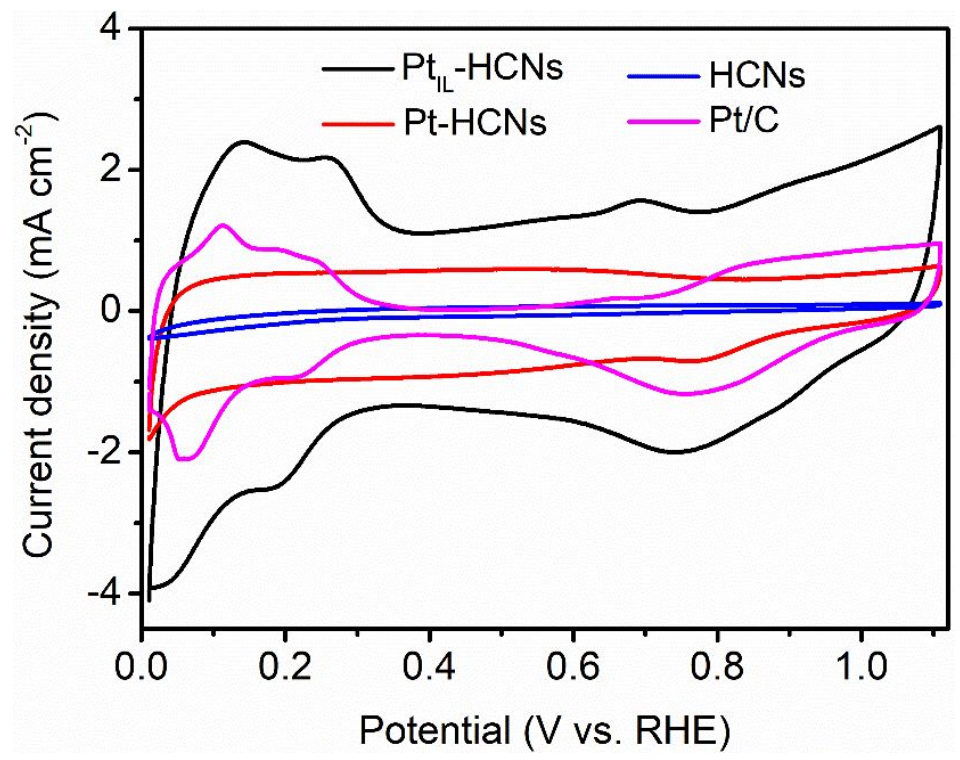

Figure S16. CV curves performed in $\mathrm{O}_{2}$-saturated $0.1 \mathrm{M} \mathrm{HClO}_{4}$ solution. 


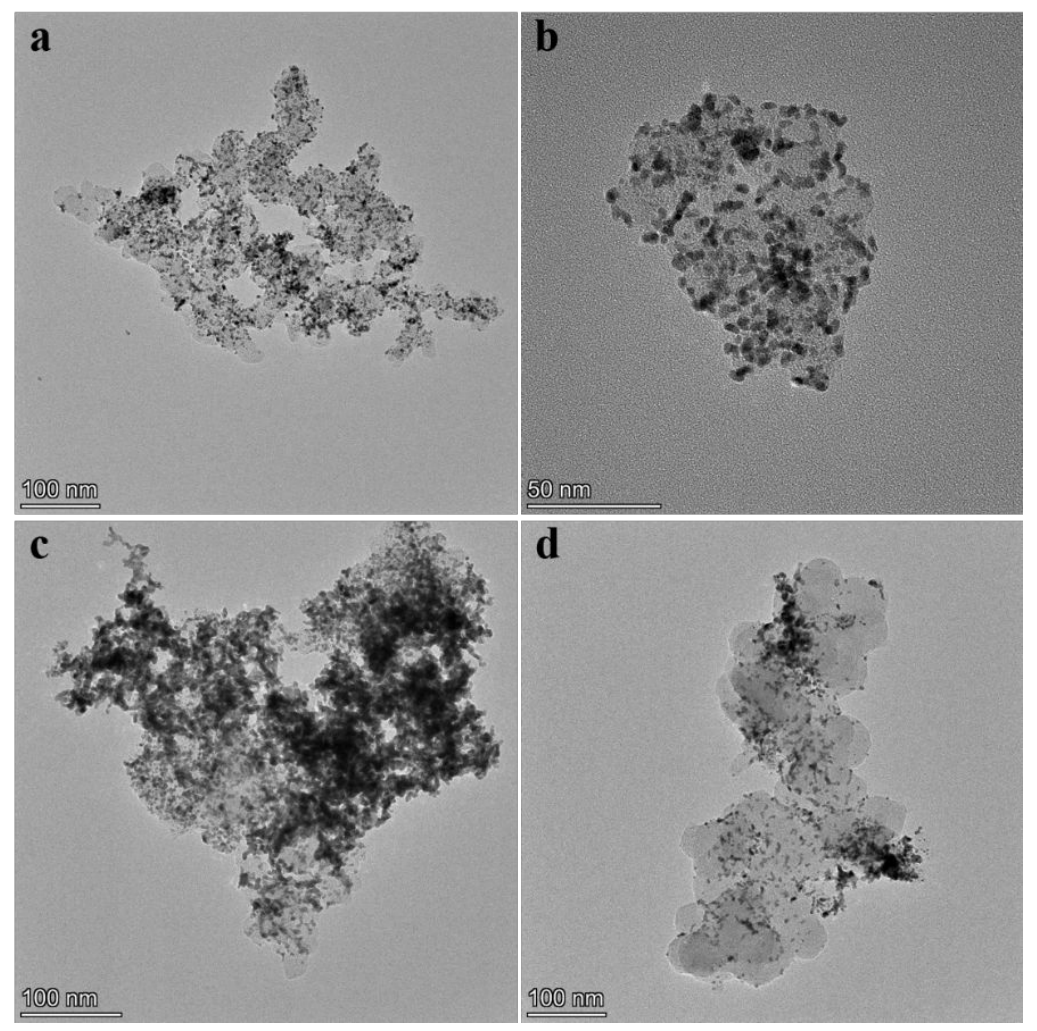

Figure S17. TEM and HRTEM images of JM Pt/C (a, b) before and (c, d) after ADT (potentiostatic polarization of 20,000s).

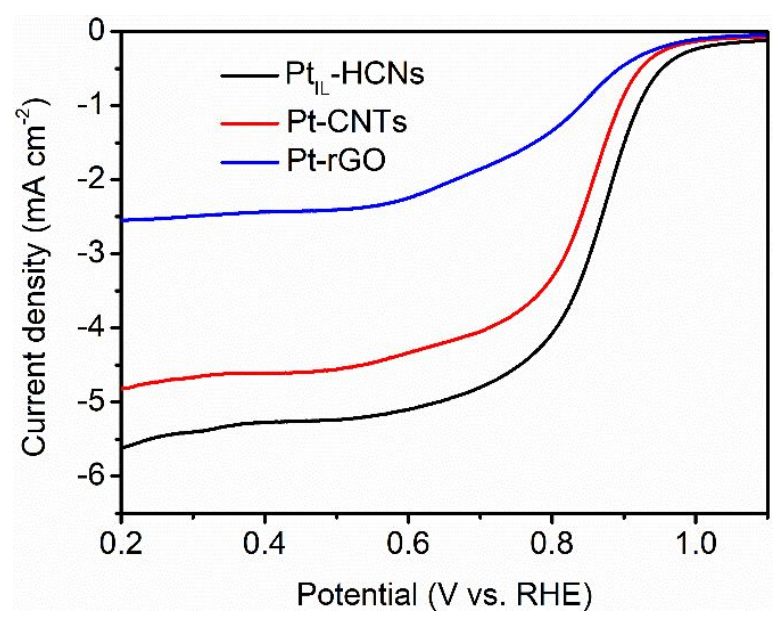

Figure S18. Polarization plots at a scan rate of $10 \mathrm{mV} \mathrm{s}^{-1}$ and a rotating speed of 1200 rpm after ADT (potentiostatic polarization of 20,000s at $0.4 \mathrm{~V}$ ). 
Table S1. Comparison of the activity of substrate-supported Pt nanoparticles electrocatalysts for ORR in $\mathrm{O}_{2}$-saturated $0.1 \mathrm{M} \mathrm{HClO}_{4}$ electrolyte.

\begin{tabular}{|c|c|c|c|c|c|c|c|c|}
\hline Samples & $\begin{array}{l}\text { Pt content } \\
\text { (wt.\%) }\end{array}$ & $\begin{array}{l}\mathrm{Pt} \text { size } \\
(\mathrm{nm})\end{array}$ & $\begin{array}{l}\text { Specific } \\
\text { surface area } \\
\left(\mathrm{m}^{2} \mathrm{~g}^{-1}\right)\end{array}$ & $\begin{array}{l}\text { Dispersed } \\
\text { states }\end{array}$ & $\begin{array}{l}\text { Half-wave } \\
\text { potential (V } \\
\text { vs. RHE) }\end{array}$ & $\begin{array}{l}\text { Mass activity at } \\
0.8 \mathrm{~V} \text { vs. } \mathrm{RHE} \\
\left(\mathrm{mA} \mathrm{mg}{ }^{-1} \mathrm{Pt}\right)\end{array}$ & $\begin{array}{l}\text { Electron } \\
\text { transfer } \\
\text { number (n) }\end{array}$ & Reference \\
\hline $\mathrm{Pt}_{\mathrm{IL}}-\mathrm{HCNs}$ & 22.0 & 2.5 & 89 & uniform & 0.88 & 475 & 4.0 & This work \\
\hline $\mathrm{Pt} / \mathrm{N}^{30} \mathrm{rGO}$ & - & 5.2 & - & agglomeration & 0.75 & - & $\sim 4.0$ & $\mathrm{~S} 1$ \\
\hline $\mathrm{Pt} / \mathrm{CB}-\mathrm{SiO}_{2} 20 \%$ & 40.0 & 4.1 & 67 & agglomeration & 0.89 & 200 & - & $\mathrm{S} 2$ \\
\hline $\mathrm{Pt}-\mathrm{TiO}_{2-\mathrm{x}} \mathrm{NSs}$ & 10.0 & 3.8 & - & agglomeration & 0.82 & 314 & $\sim 4$ & $\mathrm{~S} 3$ \\
\hline $\mathrm{Pt}_{\text {outside }} / \mathrm{KJ} 600$ & 27.8 & 2.4 & 860 & agglomeration & $\sim 0.88$ & 558 & $\sim 4$ & S4 \\
\hline $\mathrm{Pt} / \mathrm{rGO}$ & 20.0 & 3.0 & - & $\begin{array}{l}\text { slight } \\
\text { agglomeration }\end{array}$ & $\sim 0.83$ & - & $\sim 4$ & S5 \\
\hline $\mathrm{Pt} / \mathrm{NG}-\mathrm{TiON}$ & 33 & 2.1 & - & agglomeration & 0.86 & 28.52 & $\sim 4$ & S6 \\
\hline rGO_PB/Pt & - & 9.0 & - & agglomeration & 0.81 & - & 3.95 & S7 \\
\hline $\mathrm{Pt} / \mathrm{NCWG}$ & - & $\sim 3$ & 122 & agglomeration & 0.82 & - & 3.93 & $\mathrm{~S} 8$ \\
\hline $\begin{array}{l}\text { C-MOF-900- } \\
\operatorname{Pt}(1150)\end{array}$ & 8.6 & $\sim 5$ & - & $\begin{array}{l}\text { slight } \\
\text { agglomeration }\end{array}$ & 0.88 & - & 3.95 & S9 \\
\hline $\mathrm{P}_{\mathrm{NS}}-\mathrm{Pt} / \mathrm{C}$ & 20 & 3.2 & - & agglomeration & 0.92 & 1000 & - & $\mathrm{S} 10$ \\
\hline Pt/UMTHS & - & 3.2 & 262 & $\begin{array}{l}\text { slight } \\
\text { agglomeration }\end{array}$ & 0.86 & - & $\sim 4$ & S11 \\
\hline $\mathrm{PtNC} / \mathrm{ZTC}$ & $10-20$ & $0.8-1.5$ & 2360 & $\begin{array}{l}\text { slight } \\
\text { agglomeration }\end{array}$ & 0.87 & 145 & - & $\mathrm{S} 12$ \\
\hline $\mathrm{Pt} / \mathrm{Fe}-\mathrm{N}-\mathrm{C}$ & 16 & 3.3 & - & $\begin{array}{l}\text { slight } \\
\text { agglomeration }\end{array}$ & 0.88 & 460 & - & S13 \\
\hline $\mathrm{Pt} / \mathrm{NbB}_{2}$ & $0.1-11.6$ & $2-3$ & 2.6 & agglomeration & 0.81 & 165 & $\sim 4$ & S14 \\
\hline $\begin{array}{l}\mathrm{H}_{2} \quad \mathrm{Pt} / \text { carbon } \\
\text { aerogel }\end{array}$ & $>4$ & 1.5 & $400-1000$ & agglomeration & 0.67 & 107 & - & S15 \\
\hline Pt/zeolite-C & 5.33 & 12.5 & 211 & agglomeration & 0.7 & - & - & S16 \\
\hline Pt-Er@PCN-900 & 8 & 5.5 & 287 & $\begin{array}{l}\text { slight } \\
\text { agglomeration }\end{array}$ & 0.93 & - & 3.9 & S17 \\
\hline $\mathrm{Pt}-\mathrm{SnO}_{2} / \mathrm{C}$ & 11.9 & 2.43 & - & agglomeration & 0.87 & 410 & - & $\mathrm{S} 18$ \\
\hline
\end{tabular}




\section{References}

[S1] Wang, W.; Zhao, X.; Shi, H.; Liu, L.; Deng, H.; Xu, Z.; Tian, F.; Miao, X. Shape Inducer-Free Polygonal Angle Platinum Nanoparticles in Graphene Oxide as Oxygen Reduction Catalyst Derived from Gamma Irradiation. J. Colloid Interf. Sci. 2020, 575, 1-15. DOI: 1016/j.jcis.2020.04.036

[S2] Islam, J.; Kim, S.-K.; Kim, K.-H.; Lee, E.; Park, G.-G. Enhanced Durability of Pt/C Catalyst by Coating Carbon Black with Silica for Oxygen Reduction Reaction. Int. J. Hydrogen Energy 2021, 46, 1133-1143. DOI: 10.1016/j.ijhydene.2020.09.194

[S3] Naik, K. M.; Higuchi, E.; Inoue, H. Two-Dimensional Oxygen-Deficient $\mathrm{TiO}_{2}$ Nanosheets-Supported Pt Nanoparticles as Durable Catalyst for Oxygen Reduction Reaction in Proton Exchange Membrane Fuel Cells. J. Power Sources 2020, 455, 227972-227982. DOI: 10.1016/j.jpowsour.2020.227972

[S4] Jauhar, A. M.; Ma, Z.; Xiao, M.; Jiang, G.; Sy, S.; Li, S.; Yu, A.; Chen, Z. SpaceConfined Catalyst Design toward Ultrafine Pt Nanoparticles with Enhanced Oxygen Reduction Activity and Durability. J. Power Sources 2020, 473, 228607227615. DOI: 10.1016/j.jpowsour.2020.228607

[S5] Kima, J.; Kim, S.-I.; Jo, S. G.; Hong, N. E.; Ye, B.; Lee, S.; Dowd, H. S.; Lee, D. H.; Lee, J. W. Enhanced Activity and Durability of Pt Nanoparticles Supported on Reduced Graphene Oxide for Oxygen Reduction Catalysts of Proton Exchange Membrane Fuel Cells. Catal. Today 2020, 352, 10-17. DOI: 10.1016/j.cattod.2019.11.016 
[S6] Park, C.; Lee, E.; Lee, G.; Tak, Y. Superior Durability and Stability of Pt Electrocatalyst on N-Doped Graphene $\mathrm{TiO}_{2}$ Hybrid Material for Oxygen Reduction Reaction and Polymer Electrolyte Membrane Fuel Cells. Appl. Catal. B-Environ. 2020, 268, 118414-118423. DOI: 10.1016/j.apcatb.2019.118414

[S7] Zakrzewska, Barbara.; Dembinska. B.; Zoladek. S.; Rutkowska, I.; Żak, Jerzy.; Stobinski, L.; Małolepszy, A.; Negro, E.; Noto, V. Di.; Kulesza, P. J.; Miecznikowski, Krzysztof. Prussian-Blue-Modified Reduced-Graphene-Oxide as Active Support for Pt Nanoparticles During Oxygen Electroreduction in Acid Medium. J. Electroanal. Chem. 2020, 875, 114347-114356. DOI: 10.1016/j.jelechem.2020.114347

[S8] Xiong, Y.; You, M.; Liu, F.; Wu, M.; Cai, C.; Ding, L.; Zhou, C.; Hu, Min.; Deng, W.; Wang, S. Pt-Decorated, Nanocarbon-Intercalated, and N-Doped Graphene with Enhanced Activity and Stability for Oxygen Reduction Reaction. ACS Appl. Energy Mater. 2020, 3, 2490-2495. DOI: 10.1021/acsaem.9b02154

[S9] Wang, C.; Wang, X.; Lai, F.; Liu, Z.; Dong, R.; Li, W.; Sun, H.; Geng, B. Pt Nanoparticles Supported on N-Doped Porous Carbon Derived from Metal-Organic Frameworks for Oxygen Reduction. ACS Appl. Nano Mater. 2020, 3, 5698-5705. DOI: 10.1021/acsanm.0c00906

[S10] Lu, B.-A.; Shen, L.-F.; Liu, J.; Zhang, Q.; Wan, L.-Y.; Morris, D. J.; Wang, R.X.; Zhou, Z.-Y.; Li, G.; Sheng, T.; Gu, L.; Zhang, P.; Tian, N.; Sun, S.-G. Structurally Disordered Phosphorus-Doped Pt as a Highly Active Electrocatalyst for an Oxygen Reduction Reaction. ACS Catal. 2021, 11, 355-363. DOI: 
10.1021/acscatal.0c03137

[S11] He, S.; Wu, C.; Sun, Z.; Liu, Y.; Hu, R.; Guan, L.; Zhan, H. Uniform Pt Nanoparticles Supported on Urchin-Like Mesoporous $\mathrm{TiO}_{2}$ Hollow Spheres as Stable Electrocatalysts for the Oxygen Reduction Reaction. Nanoscale 2020, 12, 10656-10663. DOI: 10.1039/c9nr10890d

[S12] Bera, R. K.; Park, H.; Ko, S. H.; Ryoo, R. Highly Dispersed Pt Nanoclusters Supported on Zeolite-Templated Carbon for the Oxygen Reduction Reaction. RSC $A d v$. 2020, 10, 32290-322905. DOI: $10.1039 / \mathrm{d} 0 \mathrm{ra} 05654 \mathrm{e}$

[S13] Xie, N.-H.; Zhang, M.; Xu, B.-Q. Engineering Pt Nanoparticles with Fe and N Codoped Carbon to Boost Oxygen Reduction Catalytic Performance in Acidic Electrolyte. Energy Technol. 2020, 8, 2000393.1-2000393.7. DOI: 10.1002/ente. 202000393

[S14] Kamitaka, Yuj.; Inaba, M.; Kodama, K.; Suzuki, N.; Seki, J.; Kosaka, S.; Kimoto, Y.; Morimoto, Y. Platinum Catalysts on Niobium Diboride Microparticles for Oxygen Reduction Reaction. Electrocatal. 2021, 12, 188-198. DOI: $10.1007 / \mathrm{s} 12678-021-00644-\mathrm{Z}$

[S15] Luo, Y.; Feng, J.; Jiang, Y.; Li, L.; Feng, J. Accessible Mesoporous Carbon Aerogel Preparation and Its Application as Ultra-Low Pt Support for Oxygen Reduction Reaction with High Catalytic Activity. J. Porous Mater. 2021, 28, 661672. DOI: $10.1007 / \mathrm{s} 10934-020-01010-\mathrm{y}$

[S16] Pérez-Díaz, P. J.; Medina-Ramírez, A.; Esquivel, I. R. G.; G. Ruiz, G.; RuizCamacho, B. Effect of X Zeolite-Carbon Composite Ratio as Support of Pt 
Nanoparticles for MOR and ORR. Ionics 2021, 27, 1813-1828. DOI: $10.1007 / \mathrm{s} 11581-021-03922-4$

[S17] Nadeem, Muhammad.; Yasin, G.; Arif, M.; Tabassum, H.; Bhatti, M. H.; Mehmood, M.; Yunus, U.; Iqbal, R.; Nguyen, T. A.; Slimani, Y.; Song, H.; Zhao, W. Highly Active Sites of Pt/Er Dispersed N-Doped Hierarchical Porous Carbon for Trifunctional Electrocatalyst. Chem Eng. J. 2021, 409, 128205-128213. DOI: 10.1016/j.cej.2020.128205

[S18] Guan, J.; Zan, Y.; Shao, R.; Niu, J.; Dou, M.; Zhu, B.; Zhang, Z.; Wang, F. Phase Segregated $\mathrm{Pt}-\mathrm{SnO}_{2} / \mathrm{C}$ Nanohybrids for Highly Efcient Oxygen Reduction Electrocatalysis. Small 2020, 16, 20205048.1-202005048.7. DOI: 10.1002/smll.202005048 